\title{
Repeatedly adopting power postures does not affect hormonal correlates of dominance and affiliative behavior
}

\author{
Hannah Metzler ${ }^{\text {Corresp., } 1,2}$, Julie Grèzes ${ }^{\text {Corresp. } 1}$ \\ 1 Laboratoire de neurosciences cognitives et computationnelles, INSERM U960, Département d'études cognitives, Ecole Normale Supérieure de Paris, PSL \\ University, Paris, France \\ 2 Sorbonne Universités, Université Pierre et Marie Curie (Paris VI), Paris, France \\ Corresponding Authors: Hannah Metzler, Julie Grèzes \\ Email address: hannahmetzler1@gmail.com, julie.grezes@ens.fr
}

Background. Adopting expansive versus constrictive postures related to high versus low levels of social power has been suggested to induce changes in testosterone and cortisol levels, and thereby to mimic hormonal correlates of dominance behavior. However, these findings have been challenged by several non-replications recently. Despite this growing body of evidence that does not support posture effects on hormone levels, the question remains as to whether repeatedly holding postures over time and/or assessing hormonal responses at different time points would yield different outcomes. The current study assesses these methodological characteristics as possible reasons for previous null-findings. Additionally, it investigates for the first time whether expansive and constrictive postures impact progesterone levels, a suggested correlate of affiliative motives and behavior. By testing effects of repeated but short posture manipulations in between the blocks of a social task while using a cover story, it further fulfills the conditions previously raised as potentially necessary for the effects to occur.

Methods. 82 male participants repeatedly adopted an expansive or constrictive posture for 2 minutes in between blocks of a task that consisted in categorizing faces based on first impressions. Saliva samples were taken at two different time points in a time window in which hormonal responses to stress, competition and other manipulations are known to be strongest.

Results. Neither testosterone and cortisol levels linked to dominance behaviors, nor progesterone levels related to affiliative tendencies, responded differently to adopting expansive as opposed to constrictive postures. The present results suggest that even repeated power posing in a context where social stimuli are task-relevant does not elicit changes in hormone levels. 
1 Repeatedly adopting power postures does not affect

2 hormonal correlates of dominance and affiliative

3 behavior

4

5 Hannah Metzler ${ }^{1}, 2$, Julie Grèzes ${ }^{1}$

6

$7 \quad{ }^{1}$ Laboratoire de neurosciences cognitives et computationnelles, INSERM U960, Département

8 d'études cognitives, Ecole Normale Supérieure de Paris, PSL University, Paris, France

92 Sorbonne Universités, Université Pierre et Marie Curie (Paris VI), Paris, France 10

11 Corresponding Authors:

12 Hannah Metzler ${ }^{1,2}$

13 Email address: hannahmetzler1@gmail.com

14

15 Julie Grèzes ${ }^{1}$

16 Email address: julie.grezes@ens.fr 


\section{Abstract}

19 Background. Adopting expansive versus constrictive postures related to high versus low levels of social power has been suggested to induce changes in testosterone and cortisol levels, and thereby to mimic hormonal correlates of dominance behavior. However, these findings have been challenged by several non-replications recently. Despite this growing body of evidence that does not support posture effects on hormone levels, the question remains as to whether repeatedly holding postures over time and/or assessing hormonal responses at different time points would yield different outcomes. The current study assesses these methodological characteristics as possible reasons for previous null-findings. Additionally, it investigates for the first time whether expansive and constrictive postures impact progesterone levels, a suggested correlate of affiliative motives and behavior. By testing effects of repeated but short posture manipulations in between the blocks of a social task while using a cover story, it further fulfills the conditions previously raised as potentially necessary for the effects to occur.

Methods. 82 male participants repeatedly adopted an expansive or constrictive posture for 2 minutes in between blocks of a task that consisted in categorizing faces based on first impressions. Saliva samples were taken at two different time points in a time window in which hormonal responses to stress, competition and other manipulations are known to be strongest.

Results. Neither testosterone and cortisol levels linked to dominance behaviors, nor progesterone levels related to affiliative tendencies, responded differently to adopting expansive as opposed to constrictive postures. The present results suggest that even repeated power posing in a context where social stimuli are task-relevant does not elicit changes in hormone levels. 


\section{Introduction}

43 Individuals' position in a social hierarchy greatly determines their response to stressful situations 44 as well as their opportunities for social contact and relationships (de Waal, 1986; Sapolsky, 2005). Because individuals' social power changes over time and across different contexts, the physiological mechanisms underlying power-related behavior need to allow flexible adaptation to new situations. Steroid hormone levels, including cortisol, testosterone and progesterone are key players in the implementation of this behavioral flexibility: not only do their baseline levels influence individuals' tendencies for certain behaviors, but their levels also change in situations that involve stress, opportunities for gaining social status or affiliating with others, or threats to social status and affiliative needs (Mehta \& Josephs, 2011; Schultheiss, 2013). Although there are complex interactions between these three steroid hormones and the behaviors they modulate (Mehta \& Josephs, 2011), cortisol is predominantly involved in the regulation of stress responses (Sapolsky, 1990), testosterone seems to mediate behaviors that serve to achieve or maintain social status (Archer, 2006; Mehta \& Josephs, 2011; Eisenegger, Haushofer \& Fehr, 2011; Nave et al., 2018), and progesterone has been suggested to contribute to the regulation of affiliative behavior (Schultheiss, Wirth \& Stanton, 2004; Wirth, 2011).

Positions of high and low power are associated with distinct endocrine profiles: whereas highranking individuals have higher baseline testosterone levels and lower cortisol levels, the reverse is observed in low-ranking individuals (Sapolsky, 1990; Virgin \& Sapolsky, 1997; Mehta \& Josephs, 2010). Building on theories of embodiment, which postulate that many aspects of cognition are shaped by representations of body actions, Carney, Cuddy and Yap (2010) assessed whether exhibiting non-verbal dominant or submissive behavior, namely expanding or constricting one's body, would induce corresponding changes in testosterone and cortisol levels. They did indeed observe an increase of testosterone and a decrease of cortisol in individuals who had adopted an expansive posture and the reverse changes in individuals who had adopted a constrictive posture. Although these findings seemed consistent with the hormonal correlates of status and power, four subsequent studies could not replicate them despite large sample sizes that ensured high statistical power in three of the replications (Ranehill et al., 2015; Ronay et al., 2016; Smith \& Apicella, 2017; Davis et al., 2017). The first replication (Ranehill et al., 2015) exhibited small but potentially crucial methodological differences with the original study (see Carney, Cuddy \& Yap, 2015). Two others attempted to improve the original study's setting by testing effects in more ecologically valid social contexts that bear implications for power, status and dominance, such as competition or public speaking (Smith \& Apicella, 2017; Davis et al., 2017). Only Ronay et al.'s study (2016) was a direct replication of the original study by Carney et al. (2010), but also observed no significant effects.

All these studies focused on only power-related behavior and hormones. Yet, there is considerable evidence that power also impacts individuals' affiliative tendencies (Magee \& Smith, 2013; Guinote, 2017). For instance, lack of power enhances motivation to connect with others (Lammers et al., 2012; Case, Conlon \& Maner, 2015) and cues of low social status have positive effects on pro-social behavior (Guinote et al., 2015). Moreover, facing threats and stressful situations can enhance affiliative motivation and behavior (Schachter, 1959; Gump \& 
85 Kulik, 1997; Dezecache, Grèzes \& Dahl, 2017), as bonding with others represents an efficient 86 coping strategy (Taylor, 2006; Dezecache, 2015). The display of constrictive and submissive 87 postures generally occurs in threatening situations and serves to appease aggressive 88 conspecifics by signaling friendly intentions (Schenkel, 1967; de Waal, 1986). Adopting 89 constrictive postures may thus be linked with affiliative tendencies. Progesterone is known to be 90 released together with cortisol in response to stress in general, but particularly social stress 91 (Wirth, 2011). It correlates with both naturally fluctuating (Schultheiss, Dargel \& Rohde, 2003) 92 and experimentally induced affiliative motivation (Schultheiss, Wirth \& Stanton, 2004; Wirth \& Schultheiss, 2006) and may promote social bonding as a coping behavior in response to stress (Wirth, 2011). The question remains as to whether adopting constrictive postures would lead to a change in salivary progesterone, thereby indicating an increase of affiliation motivation.

Although expansive and constrictive postures' effects on cortisol and testosterone were not replicated, their effect on feelings of power and control, also originally reported by Carney et al. (2010), has been confirmed by a meta-analysis (Gronau et al., 2017) of six pre-registered and highly powered studies (Ronay et al., 2016; Bombari, Mast \& Pulfrey, 2017; Jackson et al., 2017; Keller, Johnson \& Harder, 2017; Klaschinski, Schnabel \& Schröder-Abé, 2017; Bailey, LaFrance \& Dovidio, 2017). On the basis of a p-curve analysis, Cuddy, Schultz and Fosse (2018) further suggested that the literature existing prior to these pre-registered studies also contains evidence for an effect on feelings of power. It should be noted that this analysis confounded significant posture effects in opposite directions (Credé, 2018), and further included several studies that did not satisfy the criteria for a robust and reproducible p-curve result (Simonsohn, Nelson \& Simmons, 2017). Nevertheless, and despite previous null-findings for hormones, the evidence for posture effects on feelings of power and other emotional and affective self-report measures in their p-curve analysis have led Cuddy et al. (2018) to call for more studies on psychophysiological outcomes. Specifically, they suggest that future experiments should apply more precise hormone methods or assess incremental effects of adopting a posture several times. Davis et al. (2017) have also raised the question of whether null-effects for hormones could be related to the timing and dose of the posture manipulation. They speculated that larger doses of posture or collection of samples at different time points after the posture could yield different outcomes. Indeed, adopting expansive postures for about 15 minutes throughout a stressful experience boosted the cortisol response to stress (Turan, 2015). This suggests that expansive postures are maladaptive in certain contexts, but also illustrates that adopting postures for longer durations may induce hormonal changes.

Altogether, it appears that additional empirical evidence is necessary to reach final conclusions about whether expansive and constrictive postures induce changes in testosterone or cortisol levels at different time points than assessed previously or when adopted for longer durations. In addition, it remains unexplored whether these postures affect hormonal correlates of affiliative behavior, such as progesterone. In 2015, before the publication of the first non-replication of the power posing effect on hormone levels (Ranehill et al., 2015), we conducted a study which we believe can contribute to the ongoing discussion about whether expansive and constrictive postures induce changes in testosterone or cortisol levels, and that additionally assessed progesterone levels for the first time. It provides an answer to questions regarding the timing of 
129 hormone measurements and the "dose" of posture recently raised by Cuddy et al. (2018) and 130 Davis et al. (2017). First, hormone levels were measured at longer time intervals after the start 131 of the posture manipulation than in previous studies. Second, incremental posture effects were 132 examined by having participants repeatedly adopt an expansive or constrictive standing posture 133 in between the blocks of a face categorization task. Additionally, participants were encouraged

The study's design met the criteria which Carney et al.'s (2015) pointed out as potentially necessary conditions for posture effects in their response to the first non-replication. Specifically, the study's procedure included (1) a credible cover story, (2) instructions delivered by an experimenter instead of a computer, (3) short time windows for adopting the postures in order to avoid discomfort, and (4) a face categorization task resembling the social filler task in the original study (Carney et al., 2010). The original social filler task consisted of forming impressions of faces without providing a response, and is commonly interpreted as a "social context" in the posture literature (Cesario \& McDonald, 2013; Carney et al., 2015). Aiming for a stronger emphasis on social aspects, we made faces task-relevant, having participants categorize them according to their implicitly assigned minimal group membership. Knowing that affective state and dominance-related personality traits can influence individuals' body posture (Weisfeld \& Beresford, 1982; Canales et al., 2010; Aviezer, Trope \& Todorov, 2012), and may therefore modulate hormonal responses to transiently adopted postures, we collected several state and trait self-report measures to check for any potentially relevant differences between the two posture groups, and control for these if necessary. In summary, the current study investigated changes in salivary testosterone, cortisol and progesterone levels in response to a repeated expansive or constrictive posture manipulation in the context of a face categorization

\section{Materials \& Methods}

156

157

158

159

160

161

162

163

164

165

166

167

168

169

170 task.

\section{Participants}

Carney et al. (2010) reported effect-sizes of $r=.34$ for testosterone and $r=.43$ cortisol. We performed a power-analysis in G-Power (Faul et al., 2007) based on the smaller one of these two effect-sizes, i.e., $r=.34$. This yielded a minimal necessary sample of $n=63$ to achieve $80 \%$ power to detect effects as large as those of Carney et al. (2010). These were the only available effect sizes for posture effects on hormone levels when we conducted our study. Given inherent biological differences in testosterone and progesterone production between men and women, analyses of these hormones need to be done separately for each sex (Stanton, 2011). Therefore, we included only male participants to achieve sufficient power with the maximum sample size possible under our feasibility constraints.

We recruited a total of 82 male participants via a participant pool mailing list and student job advertisement websites. Participants were between 17 and 32 years old, reported not to be regular smokers or under medical treatment, and to not have a history of endocrine illness, neurological and psychiatric disorders, or dependency to alcohol or other drugs. All participants 
171 provided written informed consent and were paid for their participation. The experimental

172

173

174

175

176

177

178

179

180

181

182

183

184

185

186

187

188

189

190

191

192

193

194

195

196

197

198

199

200

201

202

203

204

205

206

207

208

209

210

211

212

protocol was approved by INSERM and licensed by the local research ethics committee (Comité de protection des personnes Ile de France III - Project CO7-28, N Eudract: 207-A01125-48) and carried out in accordance with the Declaration of Helsinki.

It should be noted that our sample size was estimated with a power-analysis based on an effect size $(r=0.34)$, which is unrealistically large given the evidence acquired since 2010. Repeating the same power analysis based on a more realistic estimate of $r=0.10$ (in line with the effect on feelings of power, see Gronau et al., 2017) yields a much larger required sample size of 779 . Given that our sample size was much smaller, we conducted a sensitivity analysis to estimate the smallest effect size we could detect with $80 \%$ power. This further allowed considering specificities of our design, such as the three time points of saliva collection. The sensitivity analysis in $\mathrm{G}^{*}$ Power for a between ( 2 groups) by within ( 3 time points) interaction with 82 participants suggested that we had $80 \%$ power to detect effect sizes larger than partial etasquared $=0.06$.

\section{Measures}

Questionnaires. For assessing potential differences between the posture groups, we administered a collection of self-report questionnaires. Because power as opposed to lack of power decreases anxiety and inhibition, increases confidence, self-esteem, and approach motivation in general (Keltner, Gruenfeld \& Anderson, 2003; Guinote, 2017), we included the French version of the State-Trait Anxiety Inventory (STAI, Spielberger, 1983), the Rosenberg Self-Esteem Scale (Vallières et Vallerand, 1990), and the BIS/BAS scales which assess the behavioral activation and inhibition systems (Caci, Deschaux \& Baylé, 2007). Participants completed the trait measures prior to the testing session in the lab, but filled out the state version of the STAI after arrival at the laboratory. In addition, questions regarding compliance with behavioral restrictions before saliva collection and the dominance scale from the International Personality Item Pool (Goldberg et al. 2006, scale representing the California Psychological Inventory: http://ipip.ori.org/newCPIKey.htm\#Dominance) were administered at the end of the experiment to avoid raising suspicion about the real purpose of the posture manipulation.

Saliva collection. We collected three saliva samples $(1 \mathrm{ml}$ each) per participant using small tubes and stored them below $-20^{\circ} \mathrm{C}$ immediately after collection. After completion of the study (duration: 51 days), they were packed in dry ice and shipped to the laboratory of Clemens Kirschbaum in Dresden, where they were analyzed with commercially available chemiluminescence immunoassays with high sensitivity (IBL International, Hamburg, Germany). For a more detailed description of the assay methods used by this laboratory, see for example Ronay et al. (2016). To exclude the possible influence of external factors on hormone levels, participants were requested to refrain from drinking alcohol and exercising intensively within 24 hours before the session, from smoking or taking medical drugs on the testing day, and from eating, drinking anything except water, and tooth brushing 1.5 hours before the session. The

PeerJ reviewing PDF | (2018:11:32670:2:0:NEW 28 Feb 2019) 
213 debriefing questionnaire after the experiment showed that they largely complied with these

214 instructions (5 exceptions for alcohol, 2 for smoking).

215

216 Procedure

217 All testing sessions took place between $13 \mathrm{~h}$ and $19 \mathrm{~h}$ to attenuate effects of diurnal variation of

218 hormone levels. Saliva samples were collected as part of another study (Metzler, 2018, see

219 Study 1 in Chapter 6) during which participants had to categorize faces into in- and outgroup

220 members while repeatedly adopting postures between task blocks. Figure 1A and B depict the

221 time course of postures, saliva samples and interleaved blocks of the face categorization task.

222

223

224

225

226

227

228

229

Upon arrival, participants signed consent forms and completed the STAI state questionnaire. Following the example of a previous study (Ratner et al., 2014), we implicitly assigned participants to one of two arbitrary groups using a traditional minimal group procedure (Tajfel, 1970). Participants' task was to choose either their in- or outgroup members from pairs of faces based on their first impression. This setting provided a credible cover story, namely that the saliva samples were collected to assess associations between face categorization and physiological indices. The cover story for the postures was that a second, unrelated project on

230

231 the impact of body posture on heart rate was conducted simultaneously. Participants would adopt the posture three times for 2 min each time in between the blocks of the face

232

233 categorization task. This supposedly served to acquire heart-rate data for a total of 6 min while avoiding discomfort from holding the same posture for too long, and offered breaks during the

234

235

236

237 visually demanding task. At this point of the instructions, approximately 15 min after arrival, participants provided a first saliva sample.

Thereafter, the female experimenter determined the posture condition using a randomizing

238

239 function and provided corresponding instructions for either the expansive $(n=42)$ or the constrictive $(n=40)$ posture. Up to the point where posture instructions were provided, i.e., at the

240

241 end of all other procedures, the experimenter was blind to participant's posture in order to minimize possible experimenter biases. She first placed electrodes on participant's wrists and

242 hooked them up to the acquisition system, and demonstratively turned it on. Next, she verbally

243 provided instructions on how to place each body part without demonstrating the posture herself. The expansive and constrictive posture involved open or closed limbs, erect or slumped upper

245

246

247 body and straight or downward head tilt, respectively (see Fig. 2). The experimenter informed participants that she would check whether they correctly adopted this standing posture each time via a camera. Depending on the participant's posture condition, she finally instructed

248

249 participants to (1) sit upright with feet apart or (2) keep back and shoulders slumped and legs parallel or crossed during the task as far as comfortable for them, which supposedly served to "stabilize" the effect of postures on heart rate. This short instruction for the sitting posture was repeated on screen at the beginning of each task block. Although allowing participants to freely adjust their posture for their own comfort during the task constitutes a less controlled posture manipulation, it ensures higher ecological validity, as it corresponds to what we typically do in everyday life. Together, the repeated 2 minute periods in which participants adopted one of two

255 standing postures, together with the encouragement of a similar, but freely adaptable sitting 
256

257

258

259

260

261

262

263

264

265

266

267

268

269

270

271

272

273

274

275

276

277

278

279

280

281

282

283

284

285

286

287

288

289

290

291

292

293

294

295

296

297

298

position during the face categorization task, added up to a "larger dose" of posture while avoiding discomfort. Participants were alone while they adopted the postures and performed the task. The experimenter only briefly re-entered the room for the collection of two more saliva samples.

In total, participants thus adopted the standing posture three times, i.e., before task block 1,3 and 5 . Saliva samples were collected before the first posture and block and after block 4 and 6 . Participants had thus adopted the posture twice before sample 2, and three times before sample 3. Median block duration was 4.58 minutes (interquartile range [3.46-6.25]) depending on participants' speed in the face categorization task. This resulted in collection of saliva samples 2 and 3 approximately 23 and 36 minutes after the first posture, respectively, although the exact timing varied between participants ( $\min .14$ minutes, max. 50). This corresponds to collection of samples 2 and 3 approximately 11 and 24 minutes after the second posture, respectively, and collection of sample 3 approximately 10 minutes after the third posture.

At the end of the experiment, participants were carefully debriefed regarding suspicions about the postures. None of them had suspected a link between the posture manipulation and the saliva samples and only one participant raised doubts about our interest in a posture effect on heart-rate. Excluding him from analyses did not affect the results.

\section{Data analysis}

Outliers were determined per time point and hormone using a conservative threshold of three times the absolute deviation from the median (Leys et al., 2013), given that mean \pm SD rules are problematic for endocrine data which are rarely normally distributed (Pollet \& Meij, 2017). First, we excluded one participant from all time points and hormones due to extreme progesterone values (around 1500 pg/ml, outside of normal range even for women, see Liening et al., 2010), clearly indicating a problem with his salivary samples. Within the remaining sample of 81 participants (age $21.36 \pm 2.78$, expansive $n=41$, constrictive $n=40$ ), there were six outliers above the median plus three absolute deviations for cortisol, seven for testosterone and nine for progesterone. Results calculated without outliers did not differ from results with the full sample (see Supplementary Table S1), i.e., the same effects yielded significant or non-significant pvalues with and without outlier exclusion.

All hormone levels were log-transformed to correct for right-skewed distributions and subjected to a mixed-effects ANOVA with posture (expansive, constrictive) as a between-subject and time $(T 1, T 2, T 3)$ as a within-subject factor. Even when the time*posture interaction was not significant, we performed between-posture t-tests on the change T1-T2 or T2-T3, to allow for comparison with previous studies with only two time points. As partial eta-squared differs between within- and between-subject designs, we additionally report generalized eta-squared as an effect-size. The latter allows for comparison between different designs, as it includes within-subject variance, and excludes variance due to other factors in the design (Lakens, 2013). All analysis were done in $R$ ( $R$ Core Team, 2018) using the packages ez, psych, latticeExtra, ggplot2 and dplyr (Wickham, 2009; Lawrence, 2016; Sarkar \& Andrews, 2016;

PeerJ reviewing PDF | (2018:11:32670:2:0:NEW 28 Feb 2019) 
299 Revelle, 2017; Wickham et al., 2017). Data and analysis scripts are available at

300 https://osf.io/3nrsy/.

301

302

303

304

305

306

307

308

309

310

311

312

313

314

315

316

317

318

319

320

321

322

323

324

325

326

327

328

329

330

331

332

333

334

335

\section{Results}

All hormone levels are reported in $\mathrm{pg} / \mathrm{ml}$. Descriptive statistics for raw levels of cortisol, testosterone and progesterone separated per posture and time point, including confidence intervals, are presented in Table 1. The results are depicted in Fig. 3. Confidence intervals reported with t-tests, which were all done on log-transformed data, are log-transformed values.

\section{Cortisol}

Cortisol levels similarly decreased over time $\left(F(2,148)=79.40, p<.001, \eta^{2}{ }_{p}=0.51, \eta^{2}{ }_{G}=0.16\right)$ in both posture groups (time ${ }^{*}$ posture: $\mathrm{F}(2,148)=1.17, \mathrm{p}=.313, \eta^{2}{ }_{p}=0.02, \eta^{2}{ }_{G}=0.00$ ), in the absence of any overall difference between the groups $\left(F(1,74)=0.32, p=.576, \eta^{2}{ }_{p}=0.00, \eta^{2}{ }_{G}=\right.$ 0.00). Both the decrease from T1 to T2, i.e., from before the first posture to after adopting the posture twice, and the decrease from T2 to T3, i.e., from after the first two postures to after the third posture, were significant (T1-T2: $\left.\mathrm{t}(75)=-10.67, \mathrm{p}<.001, \mathrm{~d}_{\mathrm{z}}=-1.22,95 \% \mathrm{Cl}[-0.48,-0.33]\right)$, T2T3: $\left.\mathrm{t}(75)=-3.78, \mathrm{p}<.001, \mathrm{~d}_{\mathrm{z}}=-.43,95 \% \mathrm{Cl}[-0.24,-0.07]\right)$. The changes T1-T2 and T2-T3 were not significantly different between postures (see Table 2). Cortisol baseline levels at T1 did not significantly differ between postures $\mathrm{t}(74)=0.95, \mathrm{p}=0.346, \mathrm{~d}=0.11,[-0.14,0.40])$.

\section{Testosterone}

Levels of testosterone also decreased throughout the experiment $\left(\mathrm{F}(2,146)=19.76, \mathrm{p}<.001, \mathrm{\eta}^{2}{ }_{p}=\right.$ $0.21, \eta^{2} G=0.03$ ) with no different changes as a function of posture (time ${ }^{*}$ posture: $F(2,146)=$ $\left.1.09, p=.340, \eta^{2}{ }_{p}=0.01, \eta^{2}{ }_{G}=0.00\right)$, and no main effect of posture $\left(F(1,73)=0.13, p=.721, \eta^{2}{ }_{p}=\right.$ $\left.0.00, \eta^{2}{ }_{G}=0.00\right)$. The decrease over time was significant from the first to the second $(\mathrm{t}(74)=-$ $\left.3.53, p=.001, d_{z}=-.41,95 \% \mathrm{Cl}[-0.15,-0.04]\right)$, as well as the second to the third time point $\left(t(74)=-3.19, p=.002, d_{z}=-.37,95 \% \mathrm{Cl}[-0.11,-0.03]\right)$. Again, the decrease was similar in both posture groups (see Table 2). Testosterone baseline levels did not differ significantly between the groups $\left(t(73)=0.83, p=0.411, d=-0.10,\left[\begin{array}{ll}-0.27 & 0.11\end{array}\right]\right)$.

\section{Progesterone}

As with the two other hormones, progesterone levels declined over time $(F(2,142)=33.07$, $p<.001, \eta^{2}{ }_{p}=0.32, \eta^{2}{ }_{G}=0.06$ ) in the same manner in both posture groups (time* posture:

$\left.F(2,142)=0.04, p=.965, \eta_{p}^{2}=0.00, \eta_{G}^{2}=0.00\right)$. There was no general difference between the two postures $\left(\mathrm{F}(1,71)=2.52, \mathrm{p}=.117, \eta^{2}{ }_{p}=0.00, \eta^{2}{ }_{G}=0.03\right)$. Declines between both pairs of time points were significant $\left(\mathrm{T} 1-\mathrm{T} 2: \mathrm{t}(72)=-4.63, \mathrm{p}<.001, \mathrm{~d}_{\mathrm{z}}=-.54,95 \% \mathrm{Cl}[-0.31,-0.12]\right.$; T2-T3: $\mathrm{t}(72)=-$ $\left.3.92, p<.001, d_{z}=-.46,95 \% \mathrm{Cl}[-0.21,-0.07]\right)$. As for the other hormones, this decrease was not different between posture groups (see Table 2). Progesterone baseline levels were not significantly different between the two postures $(t(71)=1.52, p=0.132, d=0.18,95 \% \mathrm{Cl}[-0.06$, 0.48]). 


\section{Self-report questionnaires}

337 Participants from the two posture groups did not rate themselves as significantly different on

338 self-esteem $(\mathrm{t}(77)=-0.73, \mathrm{p}=.469, \mathrm{~d}=-0.08,95 \% \mathrm{Cl}[-2.89,1.34])$, trait anxiety $(\mathrm{t}(77)=0.02$,

$339 \mathrm{p}=.99, \mathrm{~d}=0.00,95 \% \mathrm{Cl}[-3.82,3.88])$, behavioral activation $(\mathrm{t}(77)=-0.15, \mathrm{p}=.88, \mathrm{~d}=-0.02,95 \% \mathrm{Cl}$

$340[-2.06,1.77])$, and inhibition $(t(77)=0.58, p=.562, d=0.07,95 \% \mathrm{Cl}[-1.05,1.92])$ prior to the

341 testing day, nor on state anxiety at the beginning $(t(79)=0.40, p=.689, d=0.045,95 \% \mathrm{Cl}[-2.68$,

$3424.04])$ or trait dominance at the end of the experiment $(t(79)=-0.90, p=.372, d=-0.10,95 \% \mathrm{Cl}[-$

$3433.37,1.28]$ ).

\section{Discussion}

345 The present experiment investigated whether adopting expansive and constrictive postures,

346 associated with high and low social power, respectively, impacts salivary levels of hormones

347 related to power, stress and affiliation. Prior to our study, only one out of five studies had

348 observed significant posture effects on testosterone and cortisol, and none had investigated

349 effects on progesterone. Several factors had been raised as explanations for why initial findings

350 of Carney et al. (2010) for testosterone and cortisol did not replicate. Our design met most of the

351

352 conditions which Carney et al. (2015) suspected to be necessary for observing postural feedback effects: first, we assessed postural effects on hormones during a face categorization

353

354

355 experiment, second, we used a cover story, third, the instructions were given by an experimenter, and fourth, participants adopted postures for maximum two minutes at a time. Moreover, following up on hypotheses raised by Cuddy et al. (2018) and Davis et al. (2017), we investigated the possibility that repeatedly holding postures over time (i.e., larger doses of posture) and/or assessing hormonal responses at longer time intervals than previous studies would induce hormonal changes.

359

360

361

Under these specific experimental conditions, neither testosterone and cortisol levels linked to dominance behaviors and stress reactions, nor progesterone levels related to affiliative

362 tendencies, changed from before to after adopting expansive or constrictive postures. Salivary

363

364 levels of testosterone, cortisol and progesterone declined from baseline to two later postposture samples, and did so similarly in the expansive and constrictive posture group. The first

365 post-posture sample captured the potential incremental effect of adopting a posture twice, at approximately 23 and 11 minutes before sample collection. The second post-posture sample reflected the effect of adopting the same posture three times, at approximately 36,24 , and 10 minutes before sample collection.

369

370

Akin to four previous studies using a single posture manipulation (Ranehill et al., 2015; Ronay et al., 2016; Smith \& Apicella, 2017; Davis et al., 2017), we did not replicate the effects reported by Carney et al. (2010). Our results add to a growing body of evidence that does not support an effect of postures on testosterone and cortisol levels. They demonstrate that even adoption of expansive and constrictive postures, for repeated but short periods of time to avoid discomfort, in between the blocks of a social task, and after providing a credible cover story, does not trigger significant hormonal changes. Thus, all the experimental characteristics listed by Carney et al. (2015) as possible reasons for null-results in Ranehill et al.'s replication (2015) were 
378

379

380

381

382

383

384

385

386

387

388

389

390

391

392

393

394

395

396

397

398

399

400

401

402

403

404

405

406

407

408

409

410

411

412

413

414

415

416

417

418

419

respected in the present study. An insufficient dose of posture as well as the collection of hormone samples at inappropriate time points after the posture manipulation (see Davis et al., 2017) therefore seem unlikely explanations for previous non-replications. The time points at which we collected saliva samples after onset of the first posture fell into the time window (20 to 40 minutes) in which experimentally induced cortisol responses are strongest (Dickerson \& Kemeny, 2004). Testosterone and progesterone responses to arousal of power and affiliation motives have been observed in a similar time window (e.g., Schultheiss, Wirth \& Stanton, 2004; Seidel et al., 2013). Still, our study does not support the conclusion that power postures elicit physiological changes associated with the experience of power and stress or the need for affiliation (Mehta \& Josephs, 2011; Wirth, 2011; Schultheiss, 2013).

Three methodological differences with previous studies merit a more detailed discussion: First, we collected three samples in total in contrast to two in all previous studies, both with a longer delay after the onset of the first posture manipulation. This procedure revealed a decline from the first to the last time point for all three hormones. This decline may either simply reflect the diurnal pattern of these hormones (Faiman \& Winter, 1971; Delfs et al., 1994; Brambilla et al., 2009; Liening et al., 2010), and/or a reduction in arousal from the start to the end of the experiment as far as cortisol is concerned. Second, we examined an exclusively male sample, whereas previous studies included mostly women (with the exception of Smith \& Apicella, 2016). If anything, this reduced variation of our dependent variables and should hence have facilitated the detection of posture effects. Moreover, in the initial study (Carney et al., 2010) and one of its replications (Ranehill et al., 2015), effects on testosterone and feelings of power were stronger in men than in women (see Credé \& Phillips, 2017). Nevertheless, we did not observe any effect in an exclusively male sample. Third, and this is a first potential limitation of our study, hormone samples were not collected at exactly the same time points for all participants as in previous studies, but after participants had finished a fixed number of blocks from the face categorization task at their own speed. Yet, the distribution of sampling time points was very similar in both posture groups and all samples were collected in a time window in which hormonal responses generally occur (Dickerson \& Kemeny, 2004; Schultheiss et al., 2012). A second limitation is that we cannot assess whether adopting the postures was a successful power manipulation, as our study did not include any behavioral measures related to power. A final limitation concerns the size of potential posture effects on hormone levels we could detect with our sample size: all effects observed in our study were smaller than the minimal effect size of $\eta_{p}^{2}=0.06$ which we could have detected with $80 \%$ power. Therefore, our sample size is too small to draw any conclusions regarding potentially existing effects below this threshold.

\section{Conclusions}

The current study assessed whether repeatedly adopting expansive and constrictive postures known as power postures induces endocrine responses that resemble the hormonal correlates of dominance and affiliative behavior. In doing so, it assessed whether larger doses of posture or collection of saliva samples at longer time intervals than previous studies would produce similar effects on testosterone and cortisol as the study by Carney et al. (2010) in contrast to previous non-replications. Second, it investigated for the first time whether adopting postures

Peer) reviewing PDF | (2018:11:32670:2:0:NEW 28 Feb 2019) 
420 elicits changes in progesterone levels, a hormone that seems to be associated with affiliative 421 motives. Participants adopted an expansive or constrictive posture three times for two minutes 422 each, in between the blocks of a face categorization task. Salivary testosterone, cortisol and 423 progesterone levels did not differ between posture groups within a time window of 14 to 50 424 minutes from the beginning of the first posture. Together with results from four previous non425 replications, our study thus makes it seem more unlikely that short-term manipulations of 426 postural expansiveness or constrictiveness elicit changes of testosterone or cortisol levels, even 427 when postures are adopted repeatedly in the context of a social task. Additionally, our results do 428 not provide evidence for an effect of power postures on progesterone levels. Although effects on 429 other outcome variables described as promising by Cuddy et al. (2018) might be reproducible, 430 the total available evidence in favor of an effect on hormones is thus currently very weak. 


\section{References}

433 Archer J. 2006. Testosterone and human aggression: an evaluation of the challenge hypothesis. $434 \quad$ Neuroscience \& Biobehavioral Reviews 30:319-345. DOI:

$435 \quad$ 10.1016/j.neubiorev.2004.12.007.

436 Bailey AH, LaFrance M, Dovidio JF. 2017. Could a woman be superman? Gender and the embodiment of power postures. Comprehensive Results in Social Psychology 0:1-22. DOI: $10.1080 / 23743603.2016 .1248079$.

Bombari D, Mast MS, Pulfrey C. 2017. Real and imagined power poses: is the physical experience necessary after all? Comprehensive Results in Social Psychology 2:44-54. DOI: 10.1080/23743603.2017.1341183.

Brambilla DJ, Matsumoto AM, Araujo AB, McKinlay JB. 2009. The effect of diurnal variation on clinical measurement of serum testosterone and other sex hormone levels in men. The Journal of Clinical Endocrinology \& Metabolism 94:907-913. DOI: 10.1210/jc.2008-1902.

445

446

447

448

449

450

451

452

453

454

455 neuroendocrine levels and risk tolerance. Psychological Science 21:1363-1368. DOI: 10.1177/0956797610383437.

Carney DR, Cuddy AJC, Yap AJ. 2015. Review and summary of research on the embodied effects of expansive (vs. contractive) nonverbal displays. Psychological Science 26:657663. DOI: $10.1177 / 0956797614566855$.

Case CR, Conlon KE, Maner JK. 2015. Affiliation-seeking among the powerless: Lacking power increases social affiliative motivation. European Journal of Social Psychology 45:378385. DOI: 10.1002/ejsp.2089.

Cesario J, McDonald MM. 2013. Bodies in context: power poses as a computation of action possibility. Social Cognition 31:260-274. 
456 Credé M. 2018. A negative effect of a contractive pose is not evidence for the positive effect of

457

458

459

460

461

462

463

464

465

466

467

468

469

470

471

472

473

474

475

476

477

478

479

480 an expansive pose: Commentary on Cuddy, Schultz, and Fosse (2018). DOI: Available at SSRN: https://ssrn.com/abstract=3198470 or http://dx.doi.org/10.2139/ssrn.3198470.

Credé M, Phillips LA. 2017. Revisiting the power pose effect: How robust are the results reported by Carney, Cuddy, and Yap (2010) to data analytic decisions? Social Psychological and Personality Science:1948550617714584. DOI: $10.1177 / 1948550617714584$

Cuddy AJC, Schultz SJ, Fosse NE. 2018. P-curving a more comprehensive body of research on postural feedback reveals clear evidential value for power-posing effects: Reply to Simmons and Simonsohn (2017). Psychological Science 29:656-666. DOI: 10.1177/0956797617746749.

Davis ML, Papini S, Rosenfield D, Roelofs K, Kolb S, Powers MB, Smits JAJ. 2017. A randomized controlled study of power posing before public speaking exposure for social anxiety disorder: No evidence for augmentative effects. Journal of Anxiety Disorders 52:1-7. DOI: 10.1016/j.janxdis.2017.09.004.

Delfs TM, Naether OG, Klein S, Leidenberger FA, Fottrell P, Zimmermann R. 1994. 24-hour profiles of salivary progesterone. Fertility and Sterility 62:960-966.

Dezecache G. 2015. Human collective reactions to threat. Wiley Interdisciplinary Reviews: Cognitive Science 6:209-219. DOI: 10.1002/wcs.1344.

Dezecache G, Grèzes J, Dahl CD. 2017. The nature and distribution of affiliative behaviour during exposure to mild threat. Royal Society Open Science 4:170265. DOI: 10.1098/rsos. 170265 .

Dickerson SS, Kemeny ME. 2004. Acute stressors and cortisol responses: A theoretical integration and synthesis of laboratory research. Psychological Bulletin 130:355-391. DOI: $10.1037 / 0033-2909.130 .3 .355$. 
481 Eisenegger C, Haushofer J, Fehr E. 2011. The role of testosterone in social interaction. Trends in Cognitive Sciences 15:263-271. DOI: 10.1016/j.tics.2011.04.008.

483 Faiman C, Winter JSD. 1971. Diurnal cycles in plasma FSH, testosterone and cortisol in men. The Journal of Clinical Endocrinology \& Metabolism 33:186-192. DOI: 10.1210/jcem-332-186.

486 Faul F, Erdfelder E, Lang A-G, Buchner A. 2007. G*Power 3: A flexible statistical power analysis program for the social, behavioral, and biomedical sciences. Behavior Research Methods 39:175-191. DOI: 10.3758/BF03193146.

Gronau QF, Erp SV, Heck DW, Cesario J, Jonas KJ, Wagenmakers E-J. 2017. A Bayesian model-averaged meta-analysis of the power pose effect with informed and default priors: the case of felt power. Comprehensive Results in Social Psychology 2:123-138. DOI: 10.1080/23743603.2017.1326760.

493

Guinote A. 2017. How power affects people: activating, wanting, and goal seeking. In: Fiske ST ed. Annual Review of Psychology, Vol 68. Palo Alto: Annual Reviews, 353-381.

495

496

497

498

499

500

501

502

503

504

505

506

Guinote A, Cotzia I, Sandhu S, Siwa P. 2015. Social status modulates prosocial behavior and egalitarianism in preschool children and adults. Proceedings of the National Academy of Sciences of the United States of America 112:731-736. DOI: 10.1073/pnas.1414550112.

Gump BB, Kulik JA. 1997. Stress, affiliation, and emotional contagion. Journal of Personality and Social Psychology 72:305-319.

Jackson B, Nault K, Richman LS, LaBelle O, Rohleder N. 2017. Does that pose become you? Testing the effect of body postures on self-concept. Comprehensive Results in Social Psychology 2:81-105. DOI: 10.1080/23743603.2017.1341178.

Keller VN, Johnson DJ, Harder JA. 2017. Meeting your inner super(wo)man: are power poses effective when taught? Comprehensive Results in Social Psychology 2:106-122. DOI: 10.1080/23743603.2017.1341186. 
507 Keltner D, Gruenfeld DH, Anderson C. 2003. Power, approach, and inhibition. Psychological 508 Review 110:265-284. DOI: 10.1037/0033-295X.110.2.265.

509 Klaschinski L, Schnabel K, Schröder-Abé M. 2017. Benefits of power posing: effects on 510 dominance and social sensitivity. Comprehensive Results in Social Psychology 2:55-67. $511 \quad$ DOI: $10.1080 / 23743603.2016 .1248080$.

512 Lakens D. 2013. Calculating and reporting effect sizes to facilitate cumulative science: a 513 practical primer for t-tests and ANOVAs. Frontiers in Psychology 4. DOI:

$514 \quad$ 10.3389/fpsyg.2013.00863.

515 Lammers J, Galinsky AD, Gordijn EH, Otten S. 2012. Power increases social distance. Social $516 \quad$ Psychological and Personality Science 3:282-290. DOI: 10.1177/1948550611418679.

517 Lawrence MA. 2016. Ez: Easy analysis and visualization of factorial experiments.

518 Leys C, Ley C, Klein O, Bernard P, Licata L. 2013. Detecting outliers: Do not use standard 519 deviation around the mean, use absolute deviation around the median. Journal of 520 Experimental Social Psychology 49:764-766. DOI: 10.1016/j.jesp.2013.03.013.

521

522

Liening SH, Stanton SJ, Saini EK, Schultheiss OC. 2010. Salivary testosterone, cortisol, and

523 progesterone: Two-week stability, interhormone correlations, and effects of time of day, menstrual cycle, and oral contraceptive use on steroid hormone levels. Physiology \& Behavior 99:8-16. DOI: 10.1016/j.physbeh.2009.10.001.

Magee JC, Smith PK. 2013. The social distance theory of power. Personality and Social Psychology Review 17:158-186. DOI: 10.1177/1088868312472732.

Mehta PH, Josephs RA. 2010. Testosterone and cortisol jointly regulate dominance: Evidence for a dual-hormone hypothesis. Hormones and Behavior 58:898-906. DOI: 10.1016/j.yhbeh.2010.08.020.

Mehta PH, Josephs RA. 2011. Social endocrinology: Hormones and social motivation. In: Dunning D ed. Social motivation. Frontiers of Social Psychology. New York, NY, US: Psychology Press, 171-189. 
533 Metzler H. 2018. The influence of bodily actions on social perception and behaviour: Assessing 534 effects of power postures. Doctoral Dissertation Thesis. Paris: Sorbonne Université.

535 Nave G, Nadler A, Dubois D, Zava D, Camerer C, Plassmann H. 2018. Single-dose

536 testosterone administration increases men's preference for status goods. Nature

537 Communications 9:2433. DOI: 10.1038/s41467-018-04923-0.

538 Pollet TV, Meij L van der. 2017. To remove or not to remove: The impact of outlier handling on 539 significance testing in testosterone data. Adaptive Human Behavior and Physiology $540 \quad 3: 43-60$. DOI: 10.1007/s40750-016-0050-z.

541 R Core Team. 2018. R: A language and environment for statistical computing. Vienna, Austria:

$542 \quad$ R Foundation for Statistical Computing.

543 Ranehill E, Dreber A, Johannesson M, Leiberg S, Sul S, Weber RA. 2015. Assessing the 544 robustness of power posing: no effect on hormones and risk tolerance in a large sample 545

546 of men and women. Psychological Science 26:653-656. DOI:

547 Ratner KG, Dotsch R, Wigboldus DHJ, van Knippenberg A, Amodio DM. 2014. Visualizing 548

549

550 minimal ingroup and outgroup faces: Implications for impressions, attitudes, and behavior. Journal of Personality and Social Psychology 106:897-911. DOI:

551 10.1037/a0036498.

Revelle W. 2017. psych: Procedures for psychological, psychometric, and personality research. 552 Evanston, Illinois: Northwestern University.

Ronay R, Tybur JM, Huijstee D van, Morssinkhof M. 2016. Embodied power, testosterone, and 554 overconfidence as a causal pathway to risk-taking. Comprehensive Results in Social Psychology 0:1-16. DOI: 10.1080/23743603.2016.1248081.

Sapolsky RM. 1990. Adrenocortical function, social rank, and personality among wild baboons. Biological Psychiatry 28:862-. DOI: 10.1016/0006-3223(90)90568-M. 
558 Sapolsky RM. 2005. The influence of social hierarchy on primate health. Science 308:648-652.

$559 \quad$ DOI: $10.1126 /$ science.1106477.

560 Sarkar D, Andrews F. 2016. latticeextra: Extra graphical utilities based on lattice.

561 Schachter S. 1959. The Psychology of Affiliation - Experimental Studies of the Sources of

$562 \quad$ Gregariousness. Stanford (Calif.): Stanford University Press.

563 Schenkel R. 1967. Submission - its features and function in wolf and dog. American Zoologist $564 \quad 7: 319-329$.

565 Schultheiss OC. 2013. The hormonal correlates of implicit motives. Social and Personality 566 Psychology Compass 7:52-65. DOI: 10.1111/spc3.12008.

567 Schultheiss OC, Dargel A, Rohde W. 2003. Implicit motives and gonadal steroid hormones:

568 effects of menstrual cycle phase, oral contraceptive use, and relationship status.

569 Hormones and Behavior 43:293-301. DOI: 10.1016/S0018-506X(03)00003-5.

570

Schultheiss OC, Schiepe A, Rawolle M, Cooper PM, Long DL, Panter AT, Rindskopf D, Sher

571 KJ. 2012. Hormone assays. APA handbook of research methods in psychology:

572 Foundations, planning, measures, and psychometrics 1:489-500.

573 Schultheiss OC, Wirth MM, Stanton SJ. 2004. Effects of affiliation and power motivation arousal 574 on salivary progesterone and testosterone. Hormones and Behavior 46:592-599. DOI:

575 10.1016/j.yhbeh.2004.07.005.

576

Seidel EM, Silani G, Metzler H, Thaler H, Lamm C, Gur RC, Kryspin-Exner I, Habel U, Derntl B. 577 2013. The impact of social exclusion vs. inclusion on subjective and hormonal reactions in females and males. Psychoneuroendocrinology 38:2925-2932. DOI:

579 10.1016/j.psyneuen.2013.07.021.

580 Simonsohn U, Nelson LD, Simmons J. 2017.[66] Outliers: Evaluating A New P-Curve Of Power 581 Poses. Available at http://datacolada.org/66 (accessed January 14, 2019). 
582 Smith KM, Apicella CL. 2017. Winners, losers, and posers: The effect of power poses on

583

584

585

586

587

588

589

590

591

592

593

594

595

596

597

598

599

600

601

602

603

604

605

606

607 testosterone and risk-taking following competition. Hormones and Behavior 92. DOI: 10.1016/j.yhbeh.2016.11.003.

Spielberger CD. 1983. Manual for the State-Trait Anxiety Inventory STAI (form Y)(“ selfevaluation questionnaire").

Stanton SJ. 2011. The essential implications of gender in human behavioral endocrinology studies. Frontiers in Behavioral Neuroscience 5. DOI: 10.3389/fnbeh.2011.00009.

Tajfel H. 1970. Experiments in intergroup discrimination. Scientific American 223:96-102.

Taylor SE. 2006. Tend and befriend: biobehavioral bases of affiliation under stress. Current Directions in Psychological Science 15:273-277. DOI: 10.1111/j.14678721.2006.00451.x.

Turan B. 2015. Is a submissive posture adaptive when being evaluated negatively? Effects on cortisol reactivity. Neuroendocrinology Letters 36:393-8.

Virgin CE, Sapolsky RM. 1997. Styles of male social behavior and their endocrine correlates among low-ranking baboons. American Journal of Primatology 42:25-39. DOI: 10.1002/(SICI)1098-2345(1997)42:1<25::AID-AJP2>3.0.CO;2-0.

de Waal FBM. 1986. The integration of dominance and social bonding in primates. The Quarterly Review of Biology 61:459-479.

Wickham H. 2009. ggplot2: Elegant graphics for data analysis. Springer-Verlag New York.

Wickham H, Francois R, Henry L, Müller K. 2017. dplyr: A grammar of data manipulation.

Wirth MM. 2011. Beyond the HPA axis: Progesterone-derived neuroactive steroids in human stress and emotion. Frontiers in endocrinology 2:1-14. DOI: 10.3389/fendo.2011.00019.

Wirth MM, Schultheiss OC. 2006. Effects of affiliation arousal (hope of closeness) and affiliation stress (fear of rejection) on progesterone and cortisol. Hormones and Behavior 50:786795. DOI: 10.1016/j.yhbeh.2006.08.003. 
Figure 1 (on next page)

Time course of the experiment and adopted body postures.

A) Time course of postures, saliva sample and task blocks. Participants adopted the same posture 3 times, before every other block of a face categorization task. Before the first posture, and after block 4 and 6, they provided a saliva sample. B) Time from the beginning of posture 1 to posture 2 and 3. C) Time from the beginning of posture 1 to saliva sample 2 and 3. 


\section{Time course}

B

Time between postures

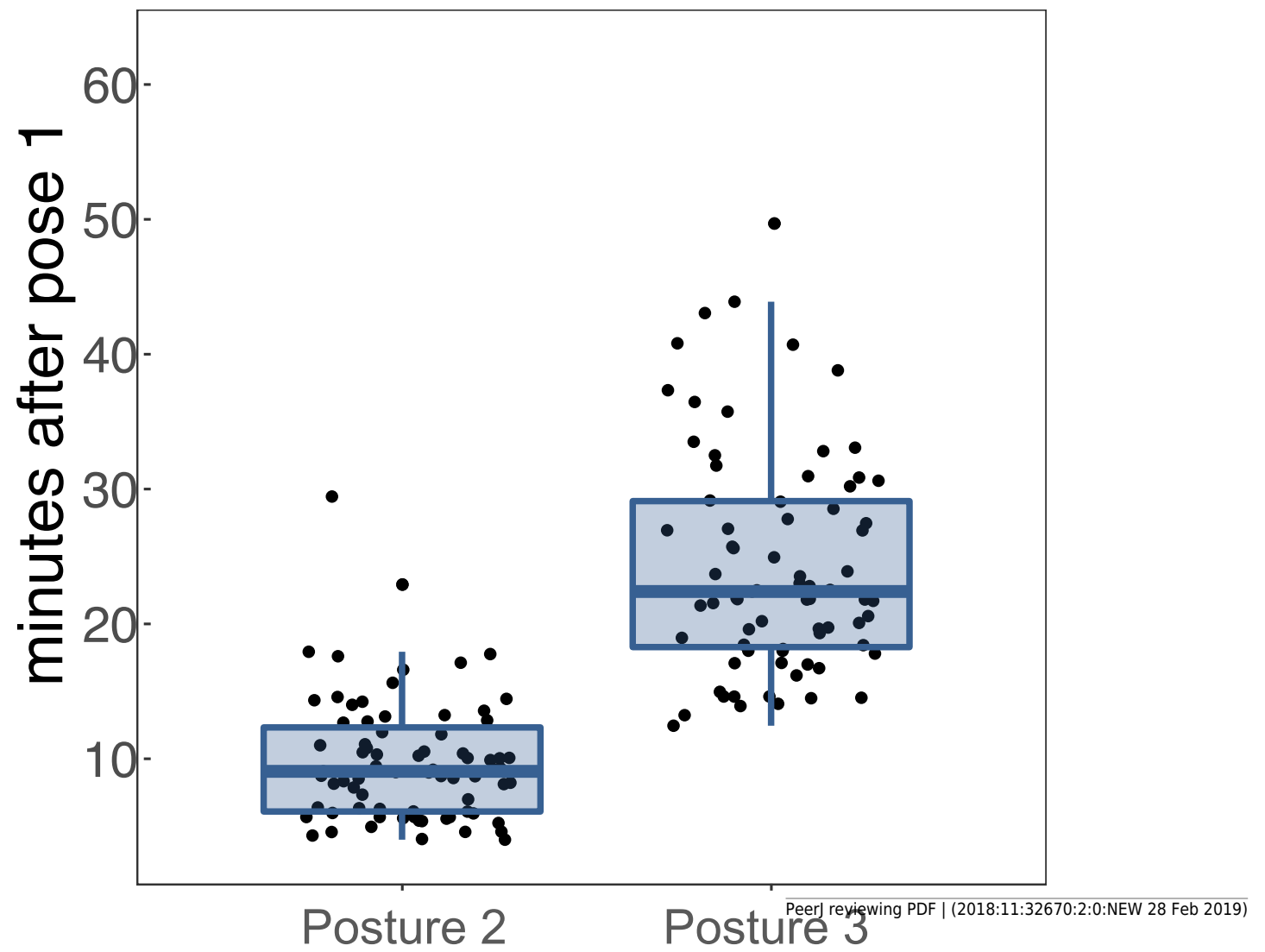

C

\section{Time between samples}

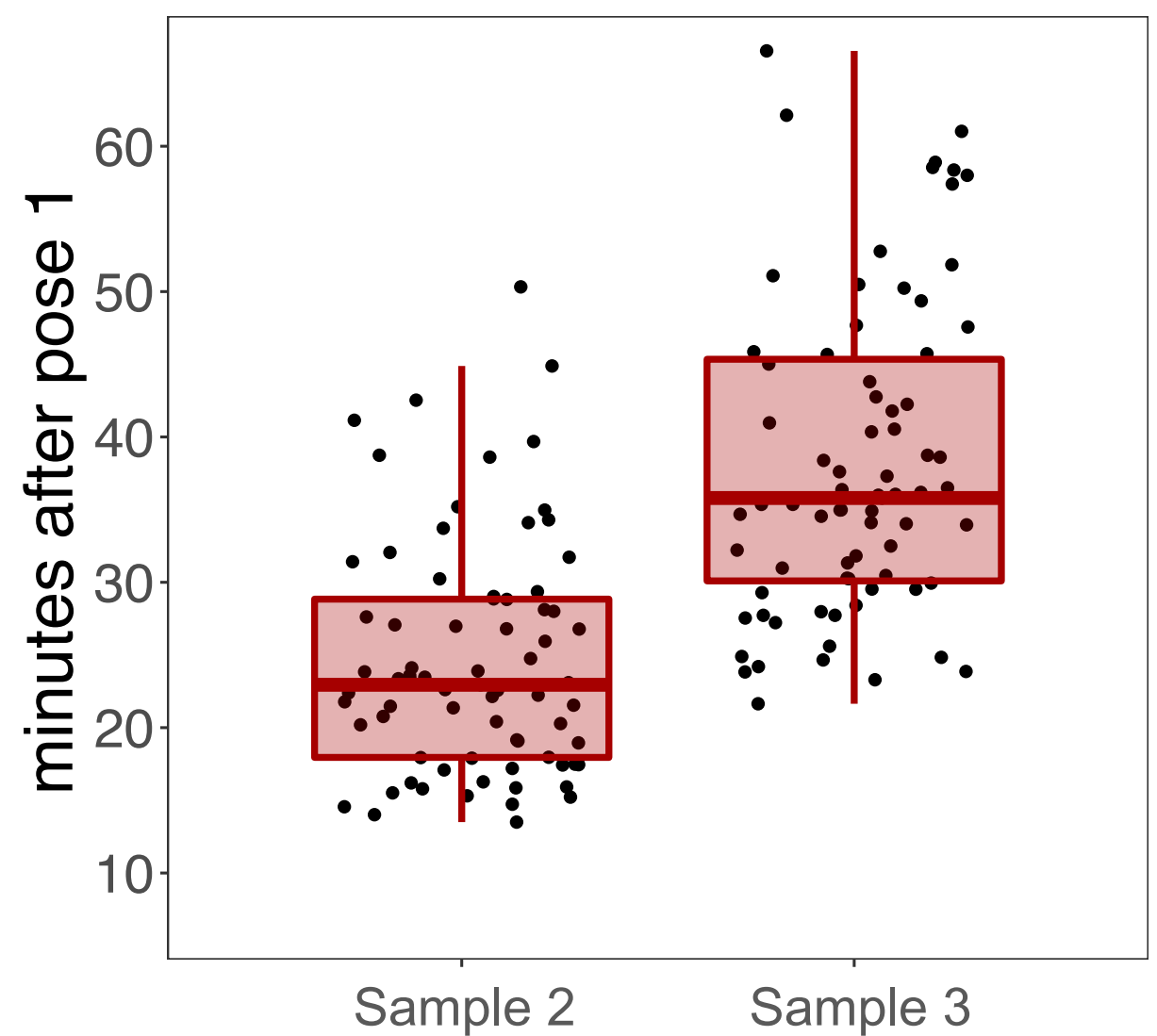

Saliva sample

Posture

Task 
Figure 2 (on next page)

Adopted postures

Postures adopted by the two experimental groups. A) Expansive posture. B) Constrictive posture. Images created by Antoine Balouka-Chadwick. 


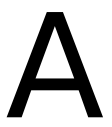

$A$

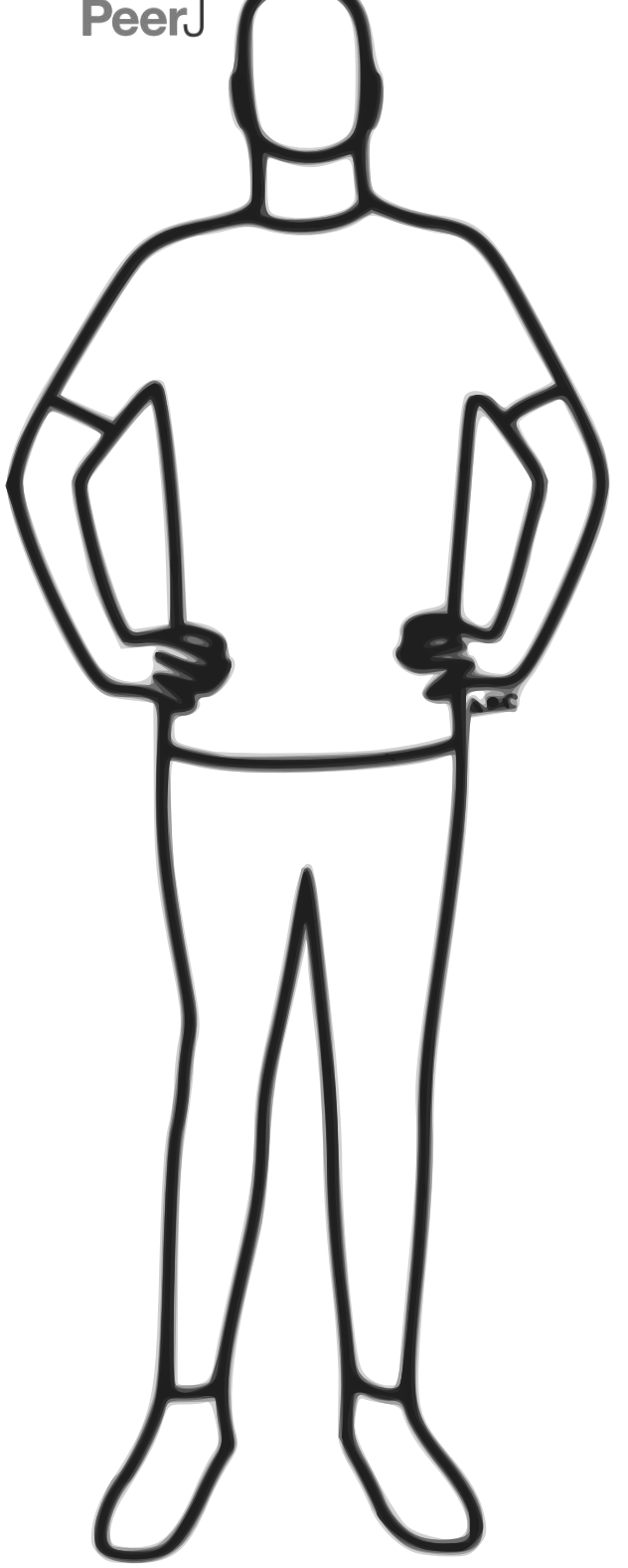

Peer] reviewing PDF | (2018:11:32670:2:0:NEW 28 Feb 2019)
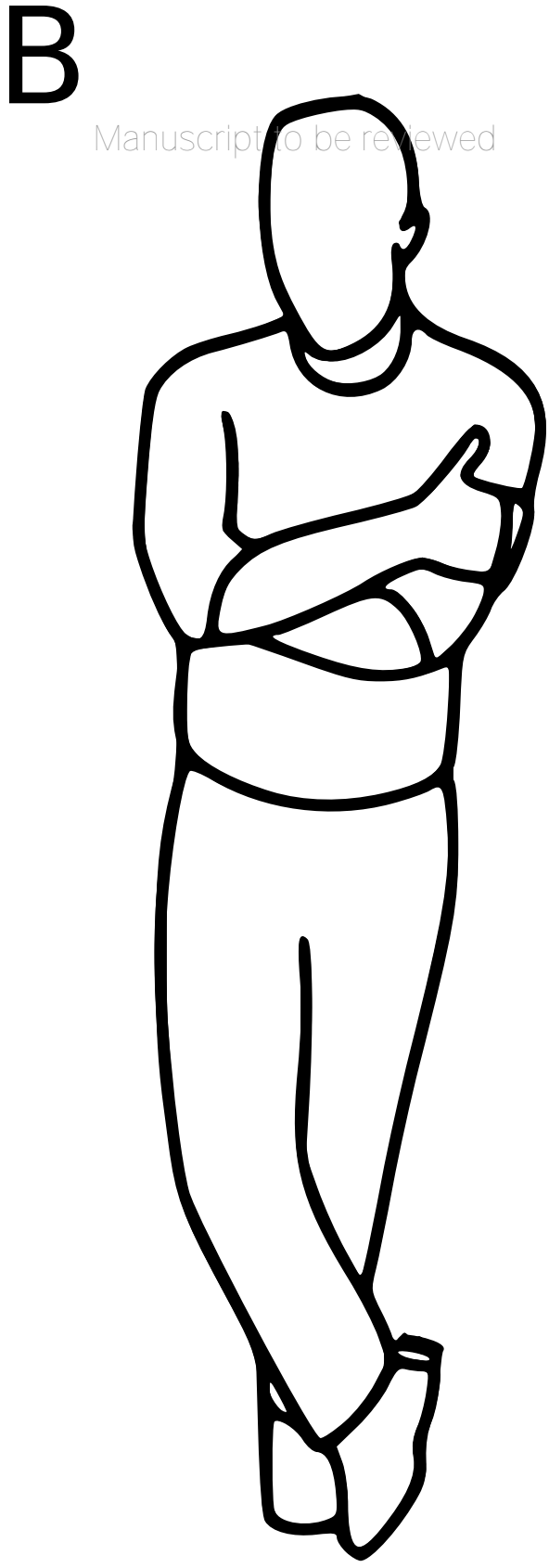

Expansive

Constrictive 
Figure 3 (on next page)

Changes in hormone levels from before to after the posture manipulation.

Means, between-subject confidence intervals and individual data points for raw values of all hormone samples $(\mathrm{C})$ in $\mathrm{pg} / \mathrm{ml}$. Sample 1 was collected before the first posture. Sample 2 and 3 reflect the effect of adopting the same posture two and three times, respectively. Asterisks indicate significance in t-tests between time points at $* * *=p<.001$ and $* * p<.01$. A) Cortisol. B) Testosterone. C) Progesterone. 
A

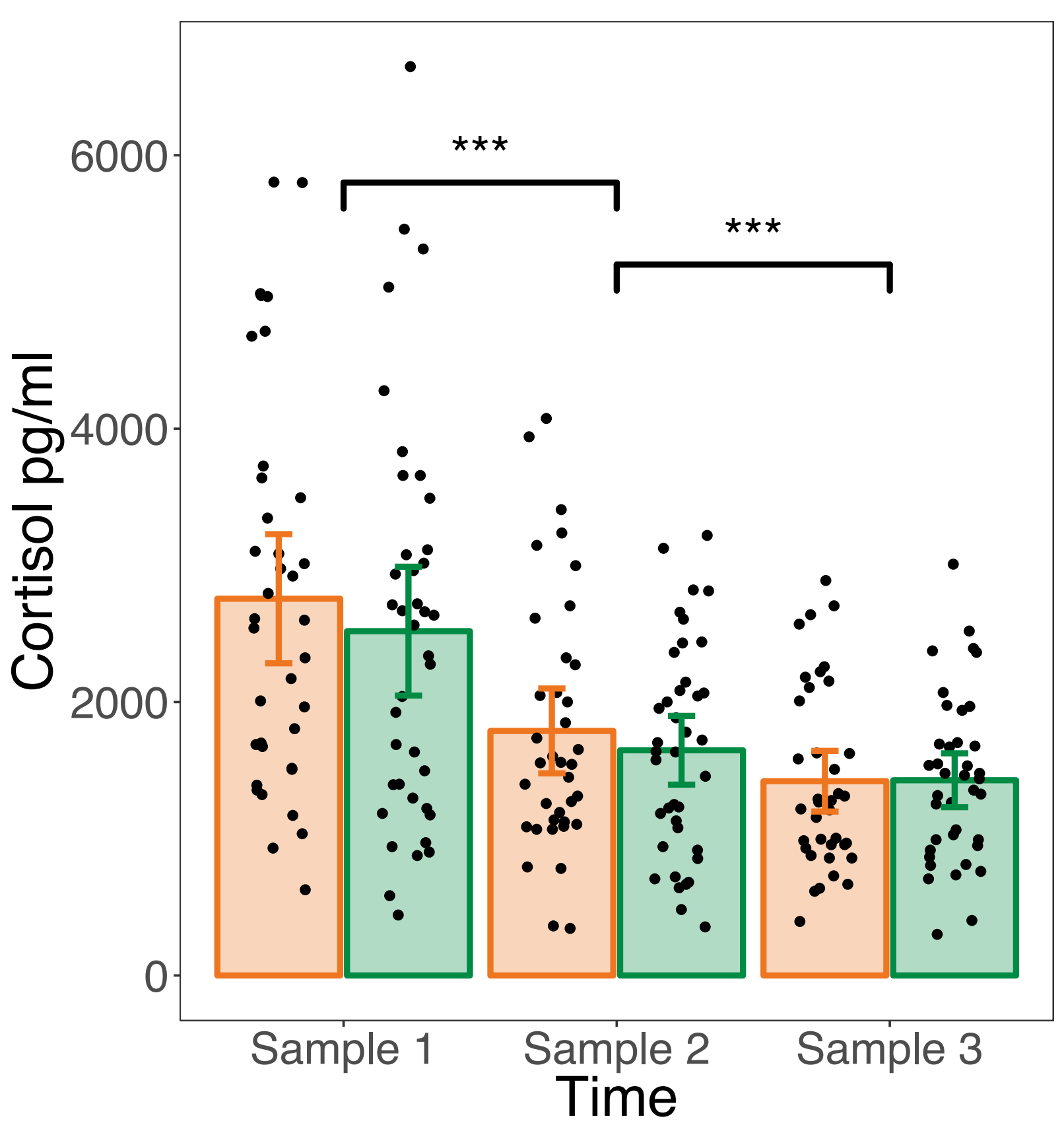

$\mathrm{B}^{\text {PeerJ }}$

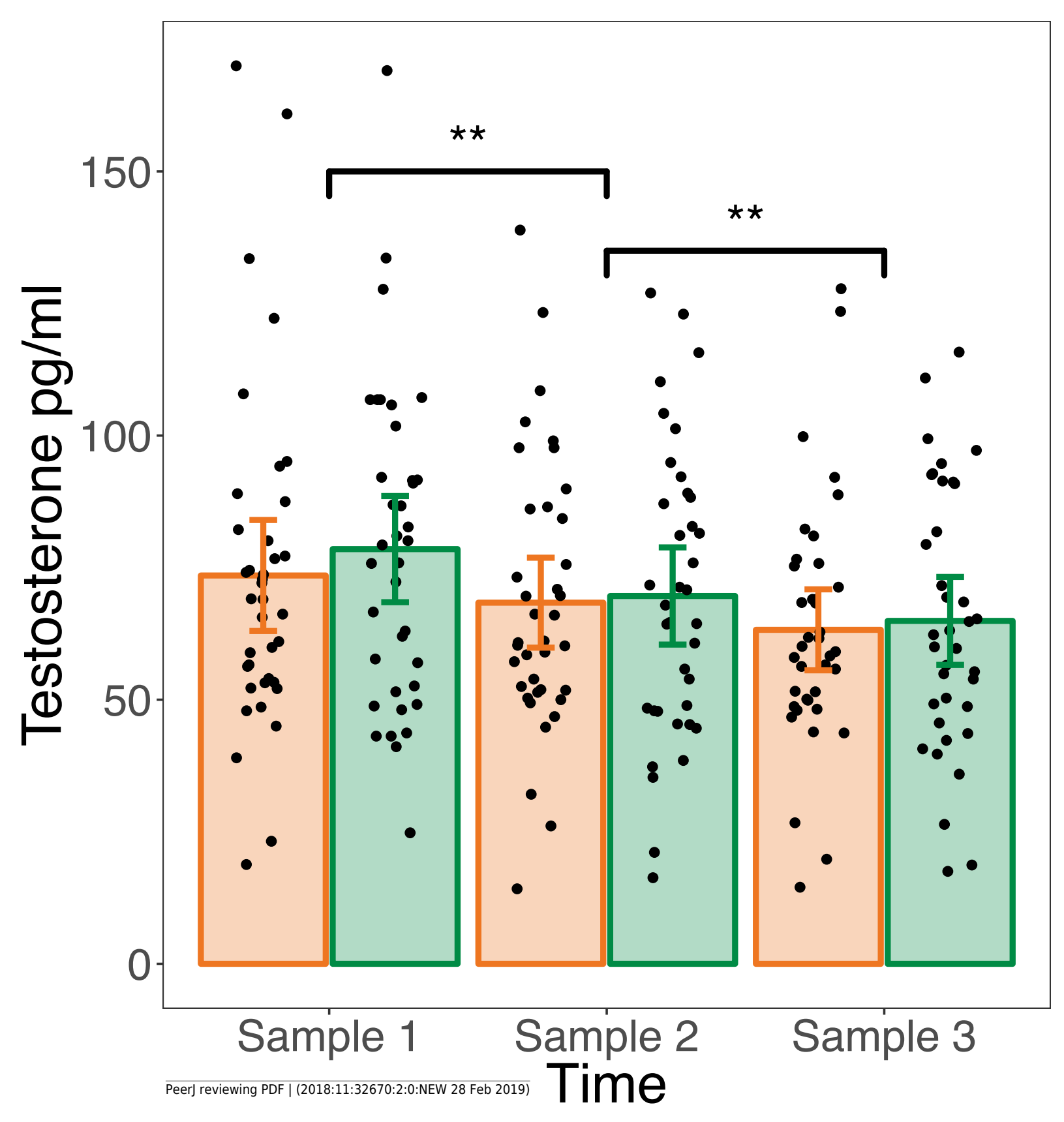

C

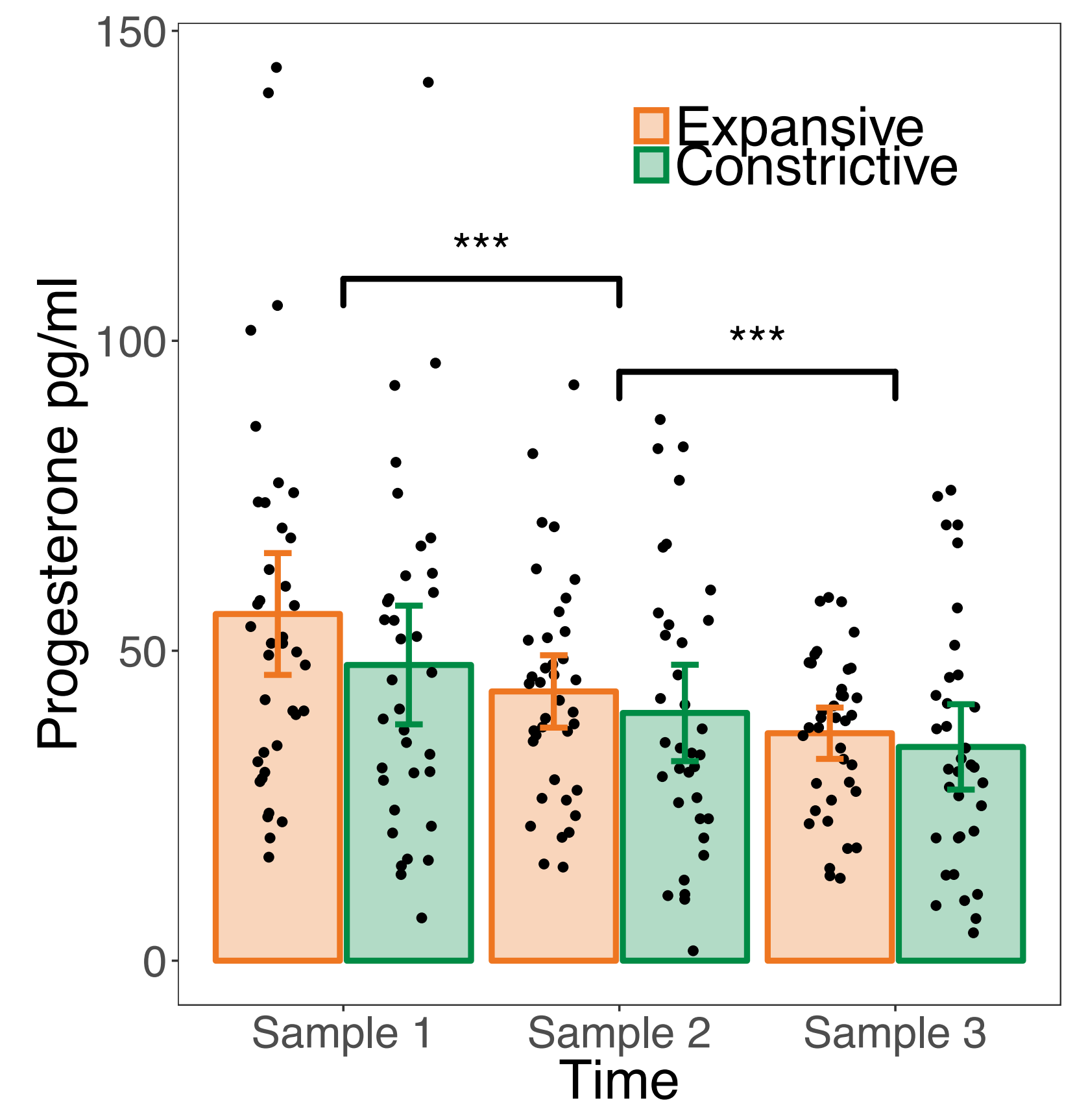




\section{Table $\mathbf{1}$ (on next page)}

Descriptive statistics for raw values of cortisol, testosterone and progesterone in samples without outliers.

Confidence intervals are between-subject to allow for between-posture comparisons. 


\begin{tabular}{|c|c|c|c|c|c|c|c|c|}
\hline & Posture & $\mathbf{N}$ & Sample & Mean & Median & SD & $95 \%$ & $\% \mathrm{Cl}$ \\
\hline \multirow[t]{6}{*}{ Cortisol pg/ml } & Expansive & 37 & 1 & 2755.88 & 2599.13 & 1416.89 & 2299.33 & 3212.44 \\
\hline & & & 2 & 1788.89 & 1555.13 & 930.94 & 1488.92 & 2088.86 \\
\hline & & & 3 & 1421.20 & 1268.75 & 665.40 & 1206.79 & 1635.60 \\
\hline & Constrictive & 39 & 1 & 2518.72 & 2562.88 & 1453.86 & 2062.43 & 2975.02 \\
\hline & & & 2 & 1647.33 & 1638.50 & 775.28 & 1404.01 & 1890.65 \\
\hline & & & 3 & 1428.06 & 1439.13 & 608.28 & 1237.16 & 1618.97 \\
\hline \multirow[t]{6}{*}{ Testosterone $\mathrm{pg} / \mathrm{ml}$} & Expansive & 38 & 1 & 73.52 & 69.05 & 31.96 & 63.35 & 83.68 \\
\hline & & & 2 & 68.37 & 60.95 & 25.90 & 60.13 & 76.60 \\
\hline & & & 3 & 63.23 & 59.60 & 23.32 & 55.81 & 70.64 \\
\hline & Constrictive & 37 & 1 & 78.51 & 79.30 & 30.14 & 68.79 & 88.22 \\
\hline & & & 2 & 69.64 & 67.90 & 27.59 & 60.74 & 78.53 \\
\hline & & & 3 & 64.92 & 62.30 & 24.97 & 56.87 & 72.96 \\
\hline \multirow[t]{6}{*}{ Progesterone $\mathrm{pg} / \mathrm{ml}$} & Expansive & 38 & 1 & 55.93 & 51.20 & 29.89 & 46.42 & 65.43 \\
\hline & & & 2 & 43.43 & 43.35 & 17.76 & 37.79 & 49.08 \\
\hline & & & 3 & 36.69 & 38.95 & 12.59 & 32.69 & 40.70 \\
\hline & Constrictive & 35 & 1 & 47.70 & 45.30 & 27.87 & 38.47 & 56.94 \\
\hline & & & 2 & 39.97 & 34.30 & 22.63 & 32.47 & 47.46 \\
\hline & & & 3 & 34.49 & 31.20 & 20.05 & 27.84 & 41.13 \\
\hline
\end{tabular}

1 


\section{Table 2 (on next page)}

T-tests and confidence intervals for hormone differences between time points in samples without outliers

T-tests were performed on log-transformed values. Confidence intervals are between-subject to allow for between-posture comparisons. 


\begin{tabular}{lcccccrrr}
\hline & $\mathbf{t}$ & $\mathbf{d f}$ & $\mathbf{p}$ & $\mathbf{d}_{\mathbf{z}}$ & $\mathbf{9 5 \%} \mathbf{C l}$ Expansive & $\mathbf{9 5 \%} \mathbf{C l}$ Constrictive \\
\hline Cortisol T2-T1 & -0.73 & 74 & 0.470 & -0.08 & -0.54 & -0.33 & -0.48 & -0.27 \\
Cortisol T3-T2 & -1.02 & 74 & 0.310 & -0.12 & -0.32 & -0.07 & -0.22 & -0.01 \\
\hline Testosterone T2-T1 & 1.32 & 73 & 0.190 & 0.15 & -0.14 & 0.02 & -0.21 & -0.06 \\
Testosterone T3-T2 & -0.21 & 73 & 0.840 & -0.02 & -0.13 & -0.02 & -0.13 & 0.00 \\
\hline Progesterone T2-T1 & 0.10 & 71 & 0.920 & 0.01 & -0.32 & -0.10 & -0.37 & -0.07 \\
Progesterone T3-T2 & -0.32 & 71 & 0.750 & -0.04 & -0.24 & -0.06 & -0.24 & -0.02 \\
\hline
\end{tabular}

1 